\title{
Marchiafava-Bignami disease: Role of neuroimaging in the diagnosis and management of acute disease
}

\author{
Shahina Bano, Shibani Mehra, Sacchida Nand Yadav, Vikas Chaudhary ${ }^{1}$ \\ Department of Radiodiagnosis, Dr. Ram Manohar Lohia Hospital and PGIMER, and ${ }^{1}$ Department of Radiodiagnosis, \\ Lady Hardinge Medical College and Associated Smt. Sucheta Kriplani and Kalawati Hospitals, Nerw Delhi - 110 001, India
}

\author{
Address for correspondence: \\ Dr. Shahina Bano, \\ Room No. 204, Doctor's Hostel, \\ Dr. Ram Manohar Lohia Hospital and \\ PGIMER, New Delhi - 110001 , India. \\ E-mail:dr_shahinaindia@yahoo.com
}

PMID: 19934570

DOI: $10.4103 / 0028-3886.57811$

\begin{abstract}
Marchiafava-Bignami disease (MBD), a rare disorder most commonly seen in patients with a history of alcohol consumption, involves progressive demyelination and subsequent necrosis of the corpus callosum. Because clinical signs are nonspecific, the role of computed tomography and magnetic resonance imaging is essential to confirm the diagnosis. Early diagnosis with imaging and prompt treatment may improve the prognosis of MBD.
\end{abstract}

Key words: Corpus callosum, choline/creatine ratio, Marchiafava-Bignami disease

\section{Introduction}

Marchiafava-Bignami disease (MBD), characterized by primary degeneration of the corpus callosum, is a rare complication of chronic alcoholism. ${ }^{[1]}$ Although nutritional deficiencies have been suspected, the cause is still unknown. The disease presentation may be acute, subacute or chronic. In the acute stage of the disease, the clinical features are highly variable and most of the cases reported have been diagnosed at autopsy. ${ }^{[2]}$ However, advances in neuroimaging has enabled early diagnosis of MBD. We describe computed tomography (CT) and magnetic resonance imaging (MRI) findings and clinical course in a patient with MBD in the early stage.

\section{Case Report}

A 32-year-old male presented to our hospital with sudden onset tonic-clonic seizures. He had a history of chronic alcohol intake and his nutritional status was very poor. On the day of admission, he was in a confused and delirious state. Few days later neurological examination revealed recent memory difficulty, dysarthic speech, disconjugate eye movements, and gait abnormalities. However, clinical features suggestive of interhemispheric disconnection were not found. Tendon reflexes were brisk and plantars were upgoing bilaterally. Laboratory tests revealed, macrocytic anemia and mild liver dysfunction. Vitamin $\mathrm{B}_{1}$ level was $20 \mathrm{ng} / \mathrm{ml}$. Cerebrospinal fluid analysis showed normal cell count and biochemistry. Values for other blood tests were within normal limits. Electroencephalogram showed bilateral diffuse slow-wave activity.

Contrast computed tomography [Figure 1] demonstrated hypodense lesion in the middle layers of the corpus callosum involving genu, body and splenium. Fast spinecho T2-weighted [Figure 2] and fluidattenuated inversion recovery [Figure 3] magnetic resonance images (MRI) revealed hyperintense lesions in the corpus callosum and these lesions were hypointense on T1-weighted images [Figure 4a]. The lesions were nonenhancing on postgadolinium images [Figure $4 \mathrm{~b}$ ]. Diffusion-weighted images [Figure 5a] revealed marked restriction with corresponding low apparent diffusion coefficient (ADC) values [Figure 5b]. On quantitative analysis, the lesion showed a low ADC value $\left(10^{-3} \mathrm{~mm}^{2} / \mathrm{s}\right)$ on the $\mathrm{ADC}$ map $=0.46 \pm 0.05$ (mean $\pm \mathrm{SD}$ ). Swelling of genu and splenium was also evident. The remaining parts of the brain, including the mammillary bodies, medial thalamus, hypothalamus, massa intermedia and periaqueductal region of the midbrain appeared normal. There was mild diffuse brain atrophy.

Based on the clinical and imaging findings, the diagnosis of MBD was made. Patient was started on intravenous 


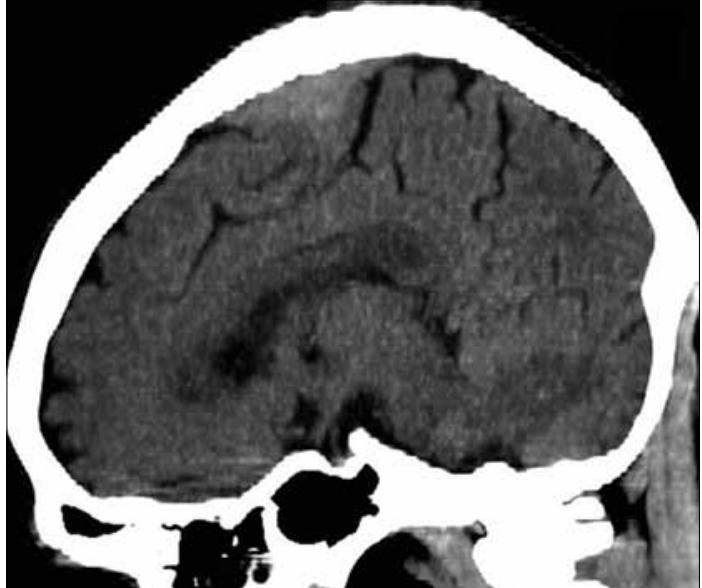

Figure 1: Unenhanced sagittal computed tomography image reveals abnormal hypodensity involving genu, body and splenium of the corpus callosum

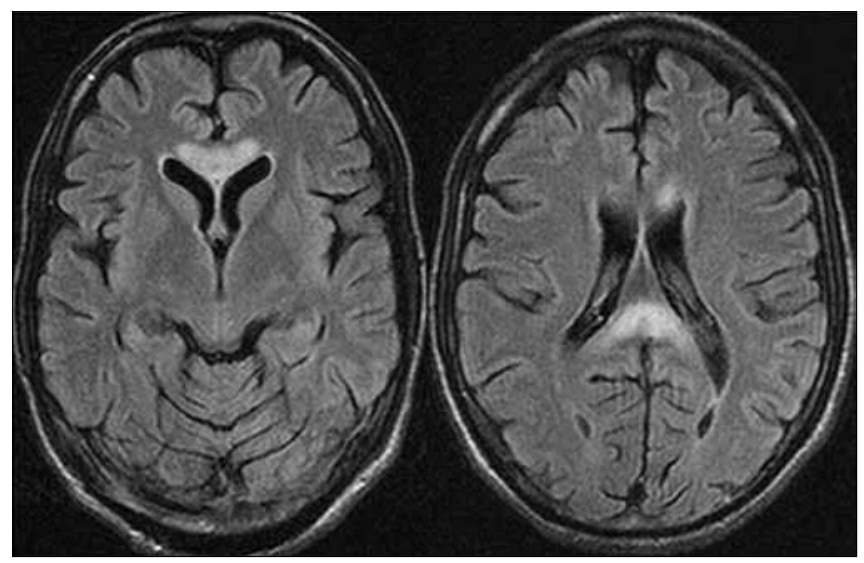

Figure 3: On axial fluid-attenuated inversion recovery image, these lesions are uniformly hyperintense due to edema and early demyelination

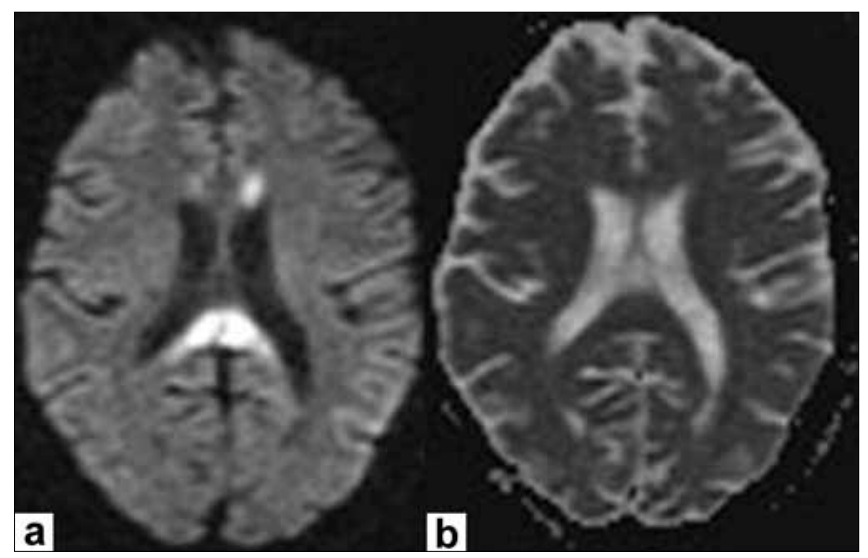

Figure 5: Axial diffusion-weighted (a) and apparent diffusion coefficient (b) image shows marked diffusion restriction in the corpus callosum

vitamin B complex and oral folate. He also received intravenous corticosteroids (250 mg methyl-prednisolone $\mathrm{q} 6 \mathrm{~h}$ ) as the attending physician believed that the possible benefit of corticosteroids would outweigh the risk. Patient had marked clinical improvement, with complete recovery over the next three weeks. T2-weighted images [Figure 6]

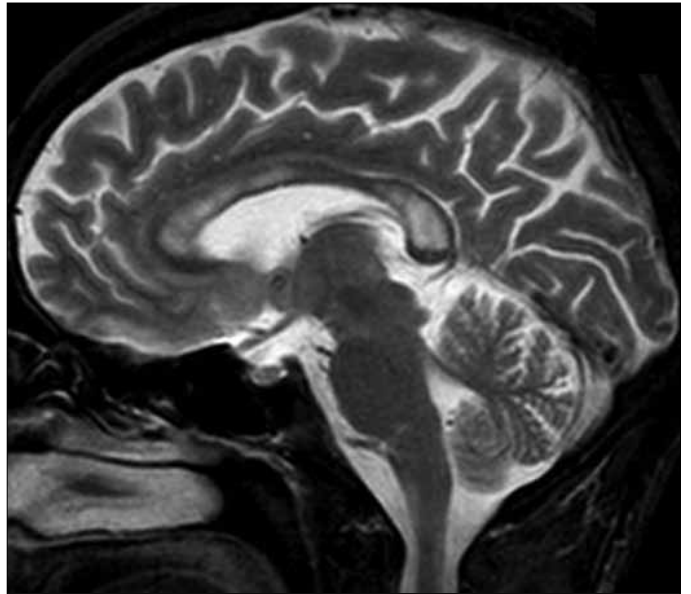

Figure 2: Sagittal $\mathrm{T}_{2}$-weighted image demonstrates hyperintense signal in the central layers of the corpus callosum with sparing of the dorsal and ventral layers producing the "sandwich sign". Mild swelling of genu and splenium of the corpus callosum is also evident

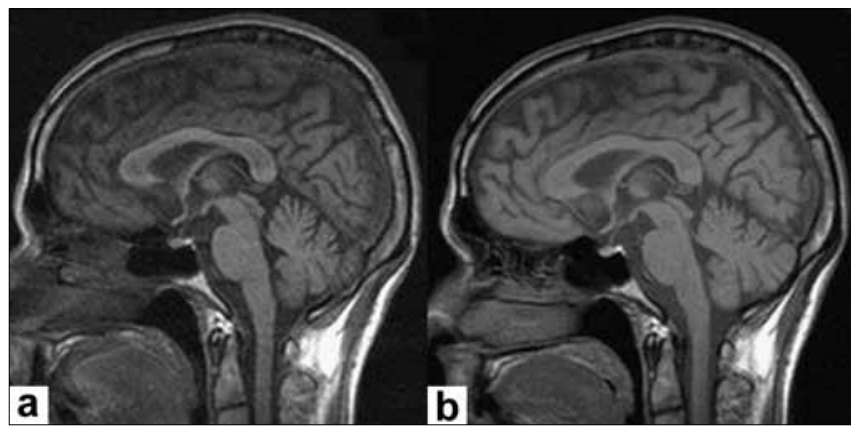

Figure 4: (a) On sagittal T1-weighted image, these lesions appear hypointense; (b) Do not enhance after administration of contrast medium

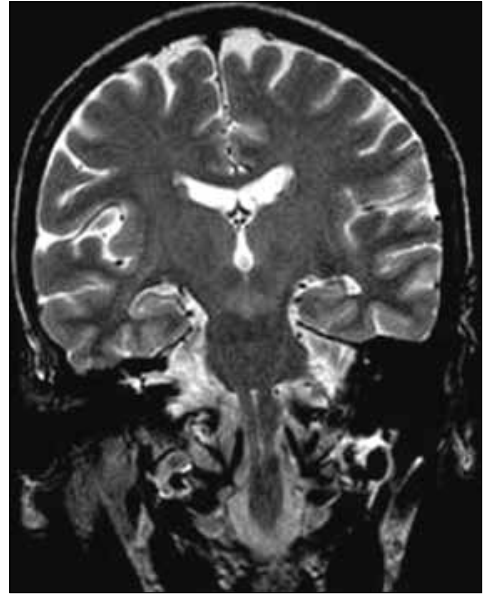

Figure 6: T2-weighted coronal image (3 weeks following treatment) reveals complete resolution of the lesion

obtained 3 weeks after the treatment revealed complete resolution of imaging abnormalities.

\section{Discussion}

The first description of MBD was in 1903 by Marchiafava and Bignami. ${ }^{[1]}$ The disease is most frequently seen in 
middle-aged or elderly alcoholic males. Rarely MBD has been reported in severely malnourished people, without history of alcoholism. It is characterized by degeneration, usually uniform, of the middle portion (middle lamina) of the myelinated fiber tracts of the corpus callosum. In some cases, the anterior and posterior commissures, the centrum semiovale and the other whitematter tracts (e.g., the long association fibers and the middle cerebral peduncles) may also be affected. Although chronic alcoholism and nutritional deficiencies have been suspected, the pathogenesis of the disease still remains controversial. ${ }^{[3]}$

Our patient had overlapping features of both Wernicke's encephalopathy and MBD. However, the vitamin $B_{1}$ level was normal and imaging did not show any characteristic features of Wernicke's encephalopathy. The radiological lesions involved the central portion of the corpus callosum, with sparing of the dorsal and ventral layer, producing the well-known "sandwich sign", the characteristic diagnostic sign of MBD. ${ }^{[4]}$ The lesions were extensive, involving genu, body and splenium, with sparing of the rostrum. In our patient the corpus callosum lesions were hypodense on CT, hypointense on T1-weighted image and hyperintense on T2-weighted/ fluid-attenuated inversion recovery (FLAIR) images. In addition there was accompaning swelling of genu and splenium. Lesions in MBD in acute or subacute phase are characterized by edema and early myelin damage. Fast spinecho T2-weighted hyperintensity of the lesion is due to both edema and myelin damage. The corpus callosum may remain hyperintense when the myelin damage is permanent and may recover to normal signal intensity with total remyelination. As lesions become chronic, cystic lesions are likely to develop. Cystic lesions are generally hyperintense on T2-weighted and hypointense on T1-weighted MR sequences. FLAIR images are even more sensitive to characterize chronic lesions. Hyperintense rim and hypointense core on FLAIR images represents gliotic rim with central necrosis, whereas uniformly hyperintense lesions indicates a combination of demyelination and edema. In acute phase the lesions are frequently larger than the lesions in the chronic phase and it is related to the associated edema. Neuropathological studies have also demonstrated edematous changes in the corpus callosum in the acute stage of MBD. ${ }^{[5,6]}$ Diffusion-weighted imaging also helps to identify demyelination, evident as areas of restricted diffusion. Unlike in stroke, however, in MBD, it is not uncommon for areas of restricted diffusion to resolve completely without apparent permanent damage. ${ }^{[7]}$ In our patient there was complete resolution of the lesions on diffusion weighted imaging following treatment.

Other imaging modalities have been used to study the pathophysiology of MBD. MR spectroscopy (MRS) revealed an increase in choline-containing compounds, resulting in increase in the choline/creatine (Cho/ $\mathrm{Cr}$ ) ratio, during the acute phase of demyelination as a result of myeline breakdown and release of phosphocholine and glycerol phosphocholine. Lactate peak is usually present during the acute/subacute phase of demyelination. Normalization of the $\mathrm{Cho} / \mathrm{Cr}$ ratio and lactate peak has been documented in patients following clinical improvement. ${ }^{[8]}$ Single photon emission computed tomography (SPECT) scans have yielded interesting pathophysiologic data in patients with MBD. In a patient with MBD, SPECT studies showed bilateral reduction in cerebral blood flow. The patient had left hemispatial neglect in addition to left-handed apraxia and agraphia. ${ }^{\left[{ }^{[9]}\right.}$ Another study has shown the importance of diffusion tensor imaging in demonstrating regional abnormalities in the corpus callosum that are not evident by conventional MRI. Fiber tracking demonstrates significant disruption of the axonal fiber bundles within the corpus callosum, most marked within the body, corresponding to the clinical finding of interhemispheric disconnection. ${ }^{[10]}$

No standardized treatment protocols have been established in MBD. However, most often patients are treated with thiamine, vitamin B-complex and folate, with good clinical recovery in many patients. Staszewski et al., treated a patient with thiamine, vitamin B-12 and folate and amantadine with improvement. ${ }^{[11]}$ Clinical improvement has been documented using high dose of corticosteroids. ${ }^{[12]}$ The available evidence suggests that an effective and aggressive early treatment is often associated with marked clinical improvement.

Other lesions involving the corpus callosum that may have similar imaging characteristics include postictal change, infarction, shearing injuries and demyelinating pathology. However, MBD can be distinguished from these disorders by the symmetry of the callosal lesions with relative sparing of the thin upper and lower edges. ${ }^{[13]}$ Tumors in this location generally have a significant mass effect and contrast enhancement and are easily distinguished from MBD. Differentiating acute MBD from Wernicke encephalopathy is not difficult because, in the latter, MRI shows abnormal signal intensity and contrast enhancement in the mamillary bodies, periaqueductal region and the walls of the third ventricle. ${ }^{[14]}$ Corpus callosal hyperintensity may also be seen in extrapontine myelinolysis (EPM), which may occur in isolation or in association with central pontine myelinolysis. The lesions in EPM are usually bilateral and symmetrical and may involve any of the subcortical structures. ${ }^{[15]}$

In summary, MBD is considered a radiological and medical emergency and early recognition is critical for 
good clinical outcome. The antimartum diagnosis of MBD mainly depends on the neuroimaging characteristics rather than the clinical features, which are often quite varied. Acute MBD may have a rapid course resulting in death. Early diagnosis and prompt institution of appropriate treatment are essential to improve the prognosis of acute MBD

\section{References}

1. Marchiafava E, Bignami A. Sopra un alterazione del corpo calloso osservata in soggetti alcoolisti. Riv Patol Nerv 1903;8:544-9.

2. Baron R, Heuser K, Marioth G. Marchiafava-Bignami disease with recovery diagnosed by CT and MRI: Demyelination affects several CNS structures. J Neurol 1989;236:364-6.

3. Berek K, Wagner M, Chemelli AP, Aichner F, Benke T. Hemispheric disconnection in Marchiafava-Bignami disease: Clinical, neuropsychological and MRI findings. J Neurol Sci 1994;123:1-2.

4. Fortman BJ, Kuszyk BS. Incidentally diagnosed Marchiafava-Bignami disease. AJR Am J Roentgenol 1999;173:1713-4.

5. Ishizaki T, Chitanondh H, Laksanavicharn U. Marchiafava-Bignami's disease. Report of the first case in an Asian. Acta neuropathol (Berl) 1970;16:187-93.

6. Kosaka K, Aoki M, Kawasaki N, Adachi Y, Konuma I, Iizuka R. A non-alcoholic Japanese patient with Wernicke's encephalopathy and Marchiafava-Bignami disease. Clin Neuropathol 1984;3:231-6.

7. Hlaihel C, Gonnaud PM, Champin S, Rousset H, Tran-Minh VA, Cotton F. Diffusion-weighted magnetic resonance imaging in Marchiafava-Bignami disease: Follow-up studies. Neuroradiology Jul 2005;47:520-4.

8. Rudkin TM, Arnold DL. Proton magnetic resonance spectroscopy for the diagnosis and management of cerebral disorders. Arch neurol 1999;56:919-26.

9. Ferracei F, Conte F, Gentile M, Candeago R, Foscolo L, Bendini M, et al. Marchiafava-Bignami disease: Computed tomographic scan, 99 mTe HMPAO-SPECT, and FLAIR MRI findings in a patient with subcortical aphasia, alexia, bilateral agraphia, and left-handed deficit of constructional ability. Arch Neurol 1999;56:107-10.

10. Sair HI, Mohamed FB, Patel S, Kanamalla US, Hershey B, Hakma Z, et al. Diffusion Tensor Imaging and Fiber-Tracking in MarchiafavaBignami Disease. J Neuroimaging 20061;16:281-5.

11. Staszewski J, Macek K, Stepien A. Reversible demyelinisation of corpus callosum in the course of Marchiafava-Bignami disease. Neurol Neurochir Pol 2006;40:156-61.

12. Kikkawa Y, Takaya Y, Niwa N. A case of Marchiafava-Bignami disease that responded to high-dose intravenous corticosteroid administration. Rinsho Shinkeigaku 2000;40:1122-5.

13. Friese SA, Bitzer M, Freudenstein D, Voigt K, Küker W. Classification of acquired lesions of the corpus callosum with MRI. Neuroradiology 2000;42:795 -802

14. Bourekas EC, Varakis K, Bruns D, Christoforidis GA, Baujan M, Slone HW, et al. Lesions of the corpus callosum: MR imaging and differential considerations in adults and children. AJR Am J Roentgenol $2002 ; 179: 251-7$.

15. Chua GC, Sitoh YY, Lim CC, Chua HC, Ng PY. MRI findings in osmotic myelinolysis. Clin Radiol 2002;57:800-6.

Accepted on 15-10-2009

Source of Support: Nil, Conflict of Interest: None declared. 